\title{
6. L'instruction des filles dans le Maghreb colonial
}

\author{
Fadma Aït Mous
}

\subsection{Introduction}

Dans le Maghreb colonial, l'instruction des femmes a constitué un enjeu politique que se disputent les autorités coloniales et les colonisés. Pour le régime colonial français, toute question touchant au statut de la 'femme musulmane' est un prétexte pour inférioriser les colonisés. Le statut dégradant de la femme maghrébine est la preuve du retard des populations soumises à la tutelle coloniale. Pour les colonisés, la femme constitue un refuge identitaire et Sliman al-Jadoui, un militant du parti du Destour cité par Daniel Rivet, déclare de façon catégorique : «La nationalité est un secret abrité dans les femmes qui demeurent la dernière fondation de notre édifice social. [...] La femme est, pour [l'homme maghrébin], un conservatoire de la nationalité vaincue. La famille s'offre comme un refuge où panser les blessures narcissiques qu'inflige à l'homme la situation coloniale » (Rivet 303). Le fait colonial a remis en question les structures sociales et culturelles des sociétés maghrébines et a eu pour effet, selon Abdellah Laroui, «non seulement de stopper l'évolution historique, mais d'obliger le colonisé à la refaire en sens inverse » (Laroui 351). C'est dire si la colonisation a contraint le Maghrébin à «se cramponner à ce que le colonisateur ne lui a pas ôté : sa foi, son sexe et sa langue » (Rivet 13).

Dans cette perspective où l'on érige la famille en rempart contre la colonisation, voire l'assimilation, les femmes se voient assigner une lourde fonction :

Les femmes feront alors partie du tabou, de l'honneur à conserver à tout prix, d'une sphère domestique que l'on s'efforce de préserver des influences extérieures néfastes. Elles sont, pour longtemps, figées dans un rôle extrêmement lourd : celui de gardiennes de la Tradition et de garantes de l'ordre 
masculin, chargées d'assurer la surcompensation des humiliations subies et déifiées pour être mieux piétinées. (Daoud 12)

Le colonisateur, en revanche, voulait leur faire jouer le rôle d'actrices du changement et de la modernisation, conformément au devoir de mission civilisatrice de la France, qui gouverne l'idéologie colonialiste de l'époque. Et « à chaque fois que la colonisation dira clairement que les femmes sont le pivot de la modernisation, qu'il faut se les acquérir pour que les sociétés maghrébines évoluent, les Maghrébines le paieront d'un recul» (Daoud 11).

$\mathrm{Au}$ prisme de l'instruction des femmes, ce texte interroge les enjeux de ces idéologies contradictoires et interposées dans les trois pays maghrébins (Maroc, Tunisie et Algérie). Ces trois pays - mis sous tutelle française de 1830 à 1962 - ont connu des processus similaires quant à l'enseignement en général et plus spécialement l'instruction des filles : une mise en place hésitante d'un enseignement scolaire colonial destiné aux Maghrébins sans s'intéresser aux Maghrébines ; un refus de la part de la population locale puis la montée progressive d'une demande scolaire à partir de l'entre-deux-guerres. Une sociologie des acteurs permet également d'identifier les acteurs (oulémas, intellectuels, jeunes nationalistes) qui ont débattu et donné ancrage à l'idée et à la nécessité de l'instruction féminine. Enfin, une analyse de discours de ces acteurs donne à voir les idéologies (colonialiste, salafiste, nationaliste) qui les animent pour justifier leurs actions à propos de la scolarisation des filles.

\subsection{Pas de politique coloniale scolaire pour les filles « indigènes »}

Le bilan de l'œuvre scolaire reste faible du point de vue quantitatif comme qualitatif. Une comparaison entre les trois pays du Maghreb démontre une situation similaire: «Les taux de scolarisation de la population musulmane étaient respectivement de 13 \% (1956) au Maroc, de 11 \% (1953) en Tunisie et de $16 \%$ (1954) en Algérie » (Akkari 227).

Commençons par souligner quelques caractéristiques des politiques scolaires coloniales relatives à l'enseignement destiné aux Maghrébins musulmans. Malgré la volonté déclarée d'instruire, il n'y a pas eu de scolarisation massive des Maghrébins en raison des faibles moyens alloués, d'obstacles et de tergiversions multiples. Cette contradiction s'expliquerait par un paradoxe inhérent à la conquête coloniale : « La politique scolaire coloniale a été très tôt 
et jusqu'à une date tardive prise au piège d'une contradiction insurmontable : scolariser, c'est acculturer mais c'est aussi éveiller les consciences et courir le risque de mettre en cause le rapport colonial» (Kadri 20).

Linstruction des filles musulmanes n’a pas été prise au sérieux par les autorités coloniales, qui n’ont guère formulé ni mis en œuvre de politique scolaire clairement destinée aux filles musulmanes maghrébines. Deux principales raisons sont le plus souvent avancées par l'administration coloniale :

Jusqu'à la Seconde Guerre mondiale, les autorités coloniales n'initièrent que peu de changements en faveur des femmes algériennes. Deux raisons furent invoquées: la première fut la promesse de la France, dans le traité de capitulation du 5 juillet 1830, de ne pas porter atteinte à la liberté des habitants de toutes classes et à leur religion, donc de ne pas intervenir dans les questions de statut personnel pourtant cruciales pour la condition féminine puisqu'elles concernaient le mariage, le divorce et les successions. La seconde raison, largement utilisée tout au long de la colonisation, fut l'opposition des musulmans à l'ingérence française sur la question de la femme. (Seferdjeli 360) $^{2}$

Au Maroc également, le traité de Fès (1912), dans son article $\mathrm{I}^{\mathrm{er}}$, avait mis en avant les réformes scolaires à mettre en place. Le premier résident général de France, H. Lyautey, «attachait de limportance à la formation des filles mais en même temps, il redoutait de bousculer les traditions et de provoquer des réactions de rejet » (Knibiehler 100).

Quantitativement parlant, le nombre de filles scolarisées dans les trois pays n'augmente qu'au lendemain de la Seconde Guerre mondiale - notamment sous la pression de la lutte nationaliste.

\subsection{Instruction des filles maghrébines: chiffres et expériences pionnières}

Comme déjà souligné, l'instruction des filles maghrébines est « un domaine laissé largement vacant par les autorités coloniales» (Seferdjeli 360). Avant le $\mathrm{XX}^{\mathrm{e}}$ siècle, l'éducation des filles était une sorte de privilège de classe sociale. Seules les femmes de l'élite pouvaient en bénéficier : l'« education for Maghribi girls tended to be restricted to women from elite families, educated within the home in urban areas, or within their family's religious brotherhood (zawiya) in rural areas » (Aït Mous et al.). 
En Algérie, il y a eu la création de quelques rares écoles sous forme d'écoleouvroir, comme celle de Mme Allix, fondée en 1845 à Alger. Le décret de 1892 qui rendait l'enseignement obligatoire pour les Algériens ne concernait que les garçons. Ce n'est qu'après 1958 que la scolarisation 'obligatoire est étendue aux filles.

Nombre de filles fréquentant l'école primaire

\begin{tabular}{|l|l|l|}
\hline & Algériennes & Européennes \\
\hline $1921-1922$ & 4455 & 38813 \\
\hline $1932-1932$ & 7260 & 49781 \\
\hline $1937-1938$ & 17286 & 58667 \\
\hline
\end{tabular}

Tableau constitué à partir des données de Seferdjeli 361.

En Tunisie existait la fameuse école «rue du Pacha», connue aussi sous le nom de l'école Millet, créée par l'épouse du résident général, Louise-Renée Millet, en 1900 à Tunis. ${ }^{3}$ Il s'agissait de la première école non missionnaire, recourant à une pédagogie moderne et subventionnée par la Résidence. Auparavant, les filles de quelques familles aristocratiques avaient eu la possibilité d'apprendre le Coran à domicile via un mou'addib (maître enseignant). Léducation des femmes était limitée à l'apprentissage du ménage et des travaux d'aiguille à la maison ou dans un atelier du quartier Dar el Maalmaa.

En 1900, le nombre de filles fréquentant l'école de Millet se limitait à 5 et s'élevait en 1903 à 30 élèves, ${ }^{4}$ sélectionnées parmi les familles des fonctionnaires tunisiens de la moyenne bourgeoisie. La directrice de l'école a dû effectuer un travail intensif de sensibilisation et user d'une grande diplomatie pour convaincre les parents des filles. Dans une lettre qu'elle a adressée au président de l'Alliance française, elle présentait ainsi ces débuts :

Le monde arabe, si fermé, ne disait rien, observait, se tenait au courant de ce qui se faisait dans notre mystérieuse école où nul Européen ne pénétrait en dehors de nos institutrices. [...] L'école ne prêche pas l'émancipation, ne touche en rien aux mœurs indigènes. On ne lutte ni contre la réclusion ni contre le voile des femmes. L'instruction et le temps pourront seuls modifier cet état de choses qui, contrairement à bien des croyances, n'est pas imposé par le Coran. (Khaddar Zangar)

Le programme de l'école comprenait l'enseignement du Coran et les éléments d'instruction arabe (lire, écrire, calculer) ainsi qu'une instruction française 
dispensée par des institutrices françaises (littérature, sciences et hygiène, histoire) et des travaux d'aiguille (broderie, couture, dentelle) (Khaddar Zangar). Ce programme a provoqué des débats au sein de la société entre certains jeunes Tunisiens, qui revendiquaient son amélioration pour aboutir au certificat d'études primaires, et d'autres qui réclamaient carrément de mettre en place un enseignement national. En 1912, lécole a déménagé dans la rue du Pacha. Elle a contribué à l'instruction de la première génération de militantes politiques et dirigeantes du mouvement des femmes en Tunisie.

Au Maroc, il faut attendre 1913 pour que s'ouvre la première école-ouvroir à Salé - conçue à partir de l'existant, à savoir les ateliers de broderie et de tapis, et confiée à une Marocaine, la mou'allima (maîtresse) Slimana (Knibiehler 100). À partir de 1917, les jeunes filles qui le souhaitaient pouvaient s'y initier au français oralement. Comme en Tunisie, les filles de l'aristocratie avaient également la possibilité d'apprendre le Coran à domicile grâce à un faqìh (maître religieux), mais aussi d'apprendre les travaux d'aiguille, toujours à domicile pour les classes supérieures ou chez la mou allima du quartier pour les autres couches sociales. D'autres centres de formation du même type suivront, incluant des séances de formation à l'hygiène et à la gestion domestique.

\subsection{Les réformistes et jeunes nationalistes maghrébins revendiquent l'instruction des filles}

Dans les trois pays maghrébins, linstruction des filles est passée d'un refus initial à une revendication de militants politiques et sociaux après la Première Guerre mondiale. Deux groupes d'acteurs ont formulé cette demande. Le premier se composait d'intellectuels formés en langue arabe et dans les mosquées-universités d'Al-Zitouna et d'Al-Qaraouiyyine. Ils faisaient partie de mouvements réformistes répandus au Maghreb à l'époque. Rappelons que ce réformisme avait pour buts de purifier la religion musulmane en la débarrassant des déviations locales et de promouvoir la langue arabe. Au Maroc, vers la fin des années 1920, Mohamed Ben Hassan el Hajoui (1874-1956), grand 'alim (intellectuel religieux) d'Al-Qaraouiyyine et ministre de l'Éducation, a donné une série de conférences pour défendre l'éducation des filles. En Algérie, c'est en 1931 qu'Abdelhamid Ben Badis (1890-1940) a fondé l'Association des oulémas musulmans algériens qui revendiquait un enseignement moderne dans les matières scientifiques, un enseignement religieux en arabe 
pour les garçons comme pour les filles et a créé des écoles « libres » à Constantine, Alger et Oran. En 1955, elles étaient au nombre de 193 et formaient 35190 enfants, dont $40 \%$ de filles (Aït Mous et al.).

En Tunisie, deux écrits rédigés à la fin $d u \mathrm{XIX}^{\mathrm{e}}$ siècle reflétaient la pensée sociale sur la condition de la femme dans l'islam. Le cheikh Ahmed Ben Dhiaf, chroniqueur et historiographe, a écrit en 1857 un opuscule intitulé Épitre de la femme (Risāla fi-l-mar'a), qui présentait la condition de la femme dans la religion musulmane. En 1897, le Cheikh Mohamed Senoussi publiait aussi un petit ouvrage, Épanouissement de la fleur (Tafattuq al-'akmām), dans un contexte polémique autour des droits de la femme dans la religion musulmane. ${ }^{5}$ Les deux se basaient sur le Coran et la sunna dans leur démarche, mais Mohamed Senoussi

n'hésite pas à s'engager en faveur de l'éducation de la femme musulmane malgré les prescriptions anciennes qui lui interdisaient par exemple l'apprentissage de l'écriture ou de la poésie. II montre, en effet, que l'éducation de la femme est importante, non seulement, pour l'accomplissement de ses devoirs religieux, mais aussi pour celui de son devoir d'épouse et de mère au foyer ayant l'obligation de bien élever ses enfants et d'aider son mari lequel, à son tour, doit satisfaire à tous les besoins moraux et matériels et participer, ainsi, à la vie du foyer. (Sraieb)

En 1920, La Tunisie martyre, un manifeste collectif critiquant le régime colonial, rédigé par un groupe d'intellectuels réformistes d'Al-Zitouna et édité par le fondateur du Destour, Abdelaziz Thâalbi (1876-1944), comprenait un chapitre sur la nécessité d'émanciper les femmes. En 1930, Tahar Haddad (18991935) écrivait Notre femme dans la charia et dans la société (Imra'atunā fi ch-charī'a wa-l-moujtama'), qui critiquait le mariage forcé, le voile, la polygamie et la répudiation. Il allait au-delà d'une simple interprétation des textes (Coran, sunna) pour placer le débat sur la condition des femmes au niveau des réalités sociales (ses rôles reproductifs, domestiques, économiques). Il considérait qu'elle était triplement prisonnière : de la maison, du voile et surtout de l'ignorance.

L'Islam n'est pas un facteur d'oppression de la femme et la société tunisienne ne pourra évoluer que lorsque la femme tunisienne se sera mise au diapason de l'évolution générale du monde, y compris et surtout du monde occidental, et qu'on lui aura permis d'agir, selon ses possibilités, au même titre que l'homme, dans tous les domaines de la vie sociale. (Sraieb) 
La question de l'éducation de la femme revêt une grande importance dans l'œuvre de T. Haddad qui en fait une exhortation :

Le devoir nous appelle, aujourd'hui plus que jamais, à sortir la femme de cet obscurantisme des siècles passés et à la considérer comme un membre vivant et un comparse égal à nous (les hommes) dans la vie, à la mesure de ses possibilités qui s'accroissent par la culture et l'enseignement. (cité par Sraieb)

Cette conception relie intrinsèquement la femme à son rôle de mère éducatrice des enfants de la nation et à ses devoirs dans l'institution familiale au sein d'une société qui évolue: "Nous voulons que la femme soit une mère capable, par la force de sa culture, de former des enfants utiles à la vie et conscients de leurs devoirs» (Sraieb).

Les jeunes nationalistes maghrébins formaient le second groupe d'acteurs ayant revendiqué l'éducation des filles. Il s'agissait de jeunes intellectuels formés soit à l'école coloniale, soit dans les écoles « libres » mises en place par les notabilités locales. Ils plaidaient pour un meilleur accès des enfants maghrébins à un système d'enseignement de qualité. Constitués en mouvements, à l'instar des «Jeunes Turcs ", ils réclamaient des réformes (amélioration des conditions de vie des populations et élargissement des droits) dans le cadre du régime colonial. Dans les programmes qu'ils présentaient aux autorités coloniales, ils demandaient de réformer le système d'enseignement pour les garçons et les filles.

Prenons l'exemple marocain du «Plan de Réformes Marocaines » élaboré par le Comité d'Action Marocaine (CAM) et présenté au sultan, au gouvernement de la République française et à la Résidence générale au Maroc en 1934. La partie consacrée à l'enseignement des filles stipulait que l'enseignement des filles marocaines devrait être basé sur la culture arabe et islamique. Les revendications concernaient la création d'écoles primaires de filles comprenant « l'enseignement obligatoire et gratuit du Coran, de l'Islam, de la langue arabe et des notions d'arithmétique, d'hygiène, de puériculture, d'art ménager et de couture » (CAM 89), alors que pour les garçons c'étaient des notions d'histoire et de géographie. Cette conception différenciée fait que les militants du CAM ont revendiqué des formations pour filles en lien uniquement avec les métiers d'institutrice, d'infirmière et de sage-femme. Une autre revendication concernait le recrutement d'institutrices musulmanes originaires d'Orient en attendant la formation des institutrices marocaines. 
En Tunisie,

les revendications du mouvement 'Jeunes Tunisiens' concernant la condition féminine portèrent essentiellement sur l'instruction des jeunes filles, une instruction donnée en langue arabe, purement musulmane, inspirée des programmes des écoles égyptiennes et turques, portant particulièrement sur les travaux manuels, la morale et l'histoire de l'Islam. Cette instruction devant être dispensée plutôt par des institutrices syriennes, 'eu égard aux affinités de langue et de coutumes' (à la différence des garçons pour qui l'instruction française ne pose pas problème), pour que les jeunes filles soient élevées selon les normes de la culture musulmane. (Bakalti 46)

Les deux types d'acteurs derrière la revendication de l'instruction des filles, intellectuels réformistes et jeunes nationalistes, partageaient une raison commune. L'instruction des filles était considérée comme particulièrement importante pour les intellectuels réformistes maghrébins, afin de fournir des épouses musulmanes instruites aux hommes musulmans instruits et de les décourager ainsi d'épouser des femmes européennes. La finalité étant de préserver la famille en tant que foyer de l'authenticité et de la transmission culturelle et par conséquent la nation. Même combat pour les jeunes nationalistes :

Ceux qui avaient été élevés dans les écoles françaises commencent alors à réclamer des compagnes un peu évoluées. Adoptant volontiers les modes européennes, ils voulaient des épouses capables d'entretenir leurs vêtements et d'accomplir de nouvelles tâches ménagères. Persuadés par leurs maîtres des bienfaits de l'hygiène, ils souhaitent que leur femme sache mieux prendre soin des enfants. Les plus instruits rêvaient d'une conjointe qui saurait lire et écrire. (Knibiehler 101)

\subsection{Conclusion}

Dans ce texte, nous avons essayé d'approcher l'instruction des filles dans le Maghreb colonial en tant que 'question', 'objet de débat' et 'catégorie d'intervention' pour les autorités coloniales et les Maghrébins. En analysant comment l'instruction des filles apparaît dans l'idéologie et les politiques coloniales d'un côté, dans les discours et les programmes des élites locales de l'autre, il ressort que, dans les deux cas, elle fait l'objet d'une instrumentalisation politique. L'éducation des filles est prônée dans le dessein de reproduire 
la nation. Les similitudes dans les processus, les profils d'acteurs et surtout leurs discours démontrent l'entrelacement des arguments utilisés communément dans les trois pays du Maghreb.

Cette analyse mérite des approfondissements et des prolongements pour voir comment cette communauté discursive autour de l'instruction des filles évolue au lendemain des indépendances du Maghreb et tenter de rendre intelligible la persistance de l'instrumentalisation de la 'question' de la femme aussi bien par les féminismes d'État que par les mouvements de société. Le paradoxe relevé à propos du rôle des femmes, à la fois gardiennes de la tradition et actrices du changement, les contraint à des renégociations permanentes de leurs droits.

\section{Bibliographie}

Ait Mous, Fadma, Kmar Bendana, et Natalya Vince. «Women in Northern African History». Oxford Research Encyclopedia of African History. Ed. Thomas Spear. Oxford University Press. Web. https://doi.org/10.1093/acr efore/9780190277734.013.685.

Akkari, Abdeljalil. «La scolarisation au Maghreb: de la construction à la consolidation des systèmes éducatifs». Carrefours de l'éducation 27 (2009): 227-244. Web. https://www.cairn.info/revue-carrefours-de-l-education-2 009-1-page-227.htm.

Bakalti, Souad, La femme tunisienne au temps de la colonisation 1881-1956. Paris : L'Harmattan, 1996.

Clancy Smith, Julia. «LÉcole Rue du Pacha, Tunis : l'enseignement de la femme arabe et 'la Plus Grande France' (1900-1914)». Clio. Histoire, femmes et sociétés 12 (2000). Web. http://journals.openedition.org/clio/186.

Comité d'Action Marocaine. Plan de réformes Marocaines. Le Caire : Imprimerie Labor, 1934.

Daoud, Zakya. Féminisme et politique au Maghreb, soixante ans de lutte. Paris : Maisonneuve et Larose, 1993.

Kadri, Aïssa. «Histoire du système d'enseignement colonial en Algérie ». La France et l'Algérie: leçons d'histoire: De lécole en situation coloniale à l'enseignement du fait colonial. Eds. Frédéric Abécassis, Gilles Boyer, Benoît Falaize, Gilbert Meynier et Michelle Zancarini-Fournel. Lyon : ENS Éditions, 2007. Web. https://books.openedition.org/enseditions/1268. 
Khaddar Zangar, Saloua. «Une école pionnière, I'École de la Rue du Pacha ». Leaders.com.tn, 12 Mai 2017. Web. https://www.leaders.com.tn/article/222 88-une-ecole-pionniere-i-ecole-de-la-rue-du-pacha.

Knibiehler, Yvonne. «Linstruction des filles au Maroc pendant le protectorat (1912-1956) ». Sextant 1 (1993) : 99-112.

Laroui, Abdellah. L'histoire du Maghreb. Un essai de synthèse. Paris : Maspero, 1970.

Rivet, Daniel. Le Maghreb à l'épreuve de la colonisation. Paris : Hachette Littératures, 2002.

Seferdjeli, Ryme. "La politique coloniale à l'égard des femmes 'musulmanes'». Histoire de l'Algérie à la période coloniale. 1830-1962. Eds. Abderrahmane Bouchène, Jean-Pierre Peyroulou, Ouanassa Siari Tengour et Sylvie Thénault. Paris : La Découverte, 2014. 359-363. Web. https://ww w.cairn.info/histoire-de-l-algerie-a-la-periode-coloniale--9782707178374 -page-359.htm.

Sraieb, Noureddine. «Islam, réformisme et condition féminine en Tunisie : Tahar Haddad (1898-1935) ». Clio. Histoire, femmes et sociétés 9 (1999). Web. h ttps://journals.openedition.org/clio/285.

\section{Notes}

1 Terme utilisé à l'époque dans les écrits et discours pour désigner les femmes 'indigènes', afin de les distinguer des femmes juives.

2 Il y a eu des tentatives de codification du statut personnel, notamment en Kabylie et dans certaines régions amazighophones au Maroc. Elles ont pris place dans une perspective assimilationniste des Berbères et non dans un but d'émancipation féminine.

3 Voir Clancy Smith (2000).

4 En 1940, les effectifs ont atteint 500 élèves. En 1945, l'école a été promue au rang de collège secondaire, le pendant du collège Sadiki pour les garçons, permettant aux jeunes filles d'accéder au baccalauréat - le sésame vers l'enseignement supérieur.

5 Voir Sraieb (1999). 